\title{
Challenges in managing children with Autism Spectrum Disorder and COVID-19: A case report
}

\author{
Shaji George, Vui Heng Chong \\ George S, Chong VH. Challenges in managing children with Autism Spectrum Disorder and COVID-19: A case report. Malays Fam Physician. \\ 2021;16(2);67-69. https://doi.org/10.51866/cr1137
}

\section{Keywords:}

Autism spectrum disorder,

autism, severe acute

respiratory distress syndrome,

coronavirus-2, SARS-CoV-2,

COVID-19, challenges

\section{Authors:}

\section{Shaji George}

(Corresponding author)

MBBS, DCH, MRCPCH [UK]

Department of Paediatrics

PMMPMHAMB Hospital, Brunei

Email: shaji.george@moh.gov.bn

\section{Chong Vui Heng}

MBChB, FAMS, FRCP Edin

Department of Medicine

PMMPMHAMB Hospital and

Division of Gastroenterology and

Hepatology, Department of Medicine RIPAS Hospital, Brunei

\begin{abstract}
Children of all ages can be affected by coronavirus disease 2019 (COVID-19), although they appear to be less affected than adults in both incidence and severity. Difficulties arise in children with co-morbid conditions such as Autism Spectrum Disorder (ASD) with regard to isolation or quarantine, investigation, and management. It can be a challenge to manage such children, be it in a national COVID center or hospital or a tertiary center. We report our experience and the challenges we faced managing two siblings with ASD and COVID-19.
\end{abstract}

\section{Introduction}

The coronavirus disease 2019 (COVID-19) pandemic has now affected almost every part of the world with over 116,166,652 confirmed cases and 2,582,528 deaths as of 7 March 2021. ${ }^{1}$ The disease mainly affects the adult population, with the highest mortality among the elderly. Children account for $1-5 \%$ of all COVID-19 cases $^{2}$ and the disease is usually mild, with fever and cough being the most common symptoms. ${ }^{2-7}$ However, managing children can be challenging, especially in children with special needs such as Autism Spectrum Disorder (ASD). Our management protocol for children required all children to be screened for infection. Mild cases were treated symptomatically. Only moderate to severe cases would be given antivirals.

\section{Case Report}

Two siblings (Sibling 1: 5 years old and Sibling 2: 12 years old) with ASD (moderate cognitive delay and intellectual disabilities) along with their mother were admitted after testing positive (reverse transcriptase polymerase chain

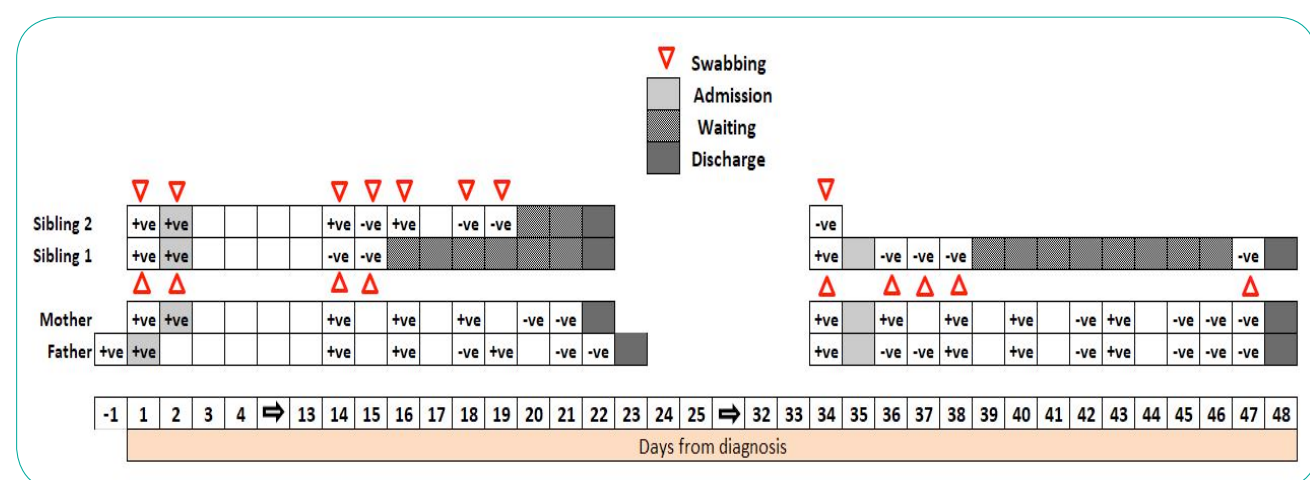

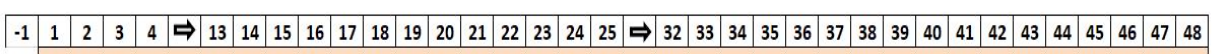
Days from diagnosis

Figure 1: Timeline of events. reaction, RT-PCR) for COVID-19, done as positive after returning from an overseas trip and was admitted a day earlier. The siblings and mother were admitted to a bay of four beds and the father was later transferred to the same bay. The children were overweight (Sibling 1 BMI $26 \mathrm{~kg} / \mathrm{m} 2$ and Sibling 2 BMI $31 \mathrm{~kg} / \mathrm{m} 2$ ). Both had mild fever and flu symptoms that had resolved on admission. Chest radiographs were normal. No blood investigations were performed at admission since the children were well. RT-PCR was repeated on admission. Figure 1 shows the timeline of events.

They were monitored using the Pediatric Early Warning Score (PEWS), which was normal throughout hospitalization. Both siblings tested negative during their exit swabs, Sibling 1 on days 14 and 15 post diagnosis, and Sibling 2 on days 18 and 19 post diagnosis. They remained in the ward as they refused to be separated from their mother. They were finally discharged together once the parents part of contact tracing. Their father tested 
tested negative. However, on the 11th day post discharge, Sibling 1 and both parents re-tested positive (re-positive) and had to be readmitted. During the readmission, Sibling 1 tested negative earlier but he had to be kept back until the parents tested negative again. All remained well on follow-up. Our post discharge protocol had mandated retesting on the 11th day for all patients.

\section{Discussion}

Autism spectrum disorder (ASD) is a neurodevelopmental disorder with persistent deficits in social communication and social interaction and restricted, repetitive patterns of behavior, interests, and activities. These children may have varying degrees of impairment such as mild, moderate, or severe. Management must be individualized according to the child's age and specific needs.

In our setting, all patients with COVID-19 are admitted for 14 days to the National Isolation Centre for treatment. Mild cases are monitored and moderate to severe cases are treated according to our treatment protocols. In managing these siblings, several challenges were encountered.

First, as part of our protocol, investigations such as nasal swabs, blood tests, and chest radiographs were performed. Swabbing for RT-PCR had to be repeated immediately after admission and again on day 14 of hospitalization (first exit swab). If a test is positive, a repeat is done 48 hours later. If the test is negative, a repeat is done 24 hours later until two consecutive negatives are obtained before discharge. Explaining the need for the swabbing and the procedure to the two children was challenging and near impossible. Although their parents tried counselling them, the siblings often refused the swab at the last moment, especially since they already had the unpleasant experience earlier. Our protocol involved taking multiple nasal swabs, which is indeed an unpleasant procedure. During swabbing, some form of restraint had to be applied, increasing the potential for injuries in general and to the nasopharynx and oral cavities in particular. After swabbing, both siblings exhibited temper tantrums, which affected their monitoring. In total, they had 17 swabs between them, including those at contact tracing; Sibling 1 had 9 and Sibling 2 had 8 swabs done. Similarly, monitoring of vital signs was an issue, particularly taking their blood pressure. Doctors and nurses in their protective personal equipment appeared less friendly, which heightened the children's anxiety levels. This is true not just for children with ASD but also for children in general. We intentionally did not do routine blood investigation unless indicated.

Second, staying in an unfamiliar hospital environment placed restrictions on their choice of play activities, food, and educational needs. Our isolation wards were adult wards without the friendly façade and play facilities that pediatric wards offer. We made arrangement for them to be admitted with their mother in a bay without any other strangers. Later, their father was moved to the same bay which helped the situation. Keeping them occupied required play materials provided by nurses, often not as suitable as their own play materials at home. This was easier for the older sibling as he kept himself occupied watching cartoons on his laptop. Although they were picky eaters, arrangements were made with the catering service to suit their needs.

Finally, discharge planning was an issue because their exit swabs became negative on different days. The siblings refused to be separated from their mother, which is true of any child, though more so in children with ASD.

Thus, we had to keep both siblings in the hospital longer than required. In our management protocol, patients are discharged for home quarantine to avoid the unnecessary risk of re-infection. Uncertainties remain regarding viral clearance, the possibility of re-infection, prolonged viral shedding, and recovery. Therefore, keeping them longer in the ward placed them at risk.

These are the main issues we faced looking after the two siblings with special needs. Fortunately, their ASD was moderate and both parents were there to manage them. It also helped that all had mild symptoms, negating the need for frequent investigations. There are several possible solutions worth considering. Mild sedation can be considered for swabbing, but there are associated risks. Use of fecal or saliva samples can be considered if available, but reliability is unknown. Special needs isolation rooms can be provided to make the hospitalization more comfortable. Work and play activities tailored to their needs can be provided to make monitoring and investigations a more pleasant experience. 
Assistance of a multi-disciplinary team will be helpful. However, with our increasing understanding of COVID-19 resulting in the quick evolution of protocols and guidelines, our management will continue to evolve as the pandemic progresses. 8 Despite this, some issues will remain in the management of patients of all ages with special needs, and future planning will need to take this into account.

\section{How does this paper make a difference to general practice?}

- COVID-19 also affects children, but manifestations are generally mild

- Management of children with special needs can present challenges

- As our understanding of COVID-19 increases, management protocols may change but some issues will remain

\section{References}

1. World Health Organization. Coronavirus disease (COVID-19) Weekly Epidemiological Update and Weekly Operational Update. Weekly epidemiological update - 9 March 2021. https://www.who.int/publications/m/ item/weekly-epidemiological-update---10march-2021 (retrieved 24 March 2021).

2. Ludwigsen JF. Systematic review of COVID-19 in children shows milder cases and a better prognosis than adults. ActaPediatric. 2020; 109:1088-95. doi: 10.1111/apa.15270.

3. Wu Z, McGoogan JM. Characteristics of and important lessons from the Coronavirus Disease 2019 (COVID-19) outbreak in China: Summary of a report of 72314 cases from The Chinese Center for Disease Control and Prevention. JAMA. 2020; 323:1239-42. doi: 10.1001/jama.2020.2648.
4. Dong Y, Mo X, Hu Y, Qi X, et al. Epidemiology of COVID-19 Among Children in China. Pediatrics. 2020;145(6):e20200702. doi: 10.1542/peds.2020-0702.

5. DeBiasi RL, Song X, Delaney M, et al. Severe COVID-19 in Children and Young Adults in the Washington, DC Metropolitan Region. J Pediatr. 2020; 223:199-203.e1. doi: 10.1016/j.jpeds.2020.05.007.

6. Mehta NS, Mytton OT, Mullins EWS, et al. SARS-CoV-2 (COVID-19): What do we know about children? A systematic review. Clin Infect Dis. 2020; 71:2469-79. doi: $10.1093 / \mathrm{cid} / \mathrm{ciaa} 556$.
7. Zimmermann P, Curtis N. Coronavirus Infections in Children Including COVID-19: An Overview of the Epidemiology, Clinical Features, Diagnosis, Treatment and Prevention Options in Children. Pediatr Infect Dis J. 2020;39:355-68. doi: 10.1097/ INF.0000000000002660.

8. Academy of Medicine Singapore. Position Statement from the National Centre for Infectious Diseases and the Chapter of Infectious Disease Physicians, Academy of Medicine, Singapore-23 May 2020. 